\title{
El mundo traicionado: traducción y falsificación en Jorge Luis Borges y en Tahar Ben Jelloun
}

\author{
DULCE MARÍA G. QUIROZ BUSTAMANTE \\ Universidad Nacional Autónoma de México
}

\section{Resumen}

En este trabajo se abordará la relación intertextual entre Jorge Luis Borges y Tahar Ben Jelloun. El escritor argentino interviene como personaje en la novela de Ben Jelloun L'enfant de sable. La inserción de Borges en esta novela contribuye a la inestabilidad del mundo narrado. En la novela de Ben Jelloun, resulta imposible asir la veracidad de la historia contada. La presencia de Borges en el relato propone una inversión de los valores de realidad y de ficción. La identidad de los personajes está en juego y el único recurso de salvación consiste en continuar contando historias, en una especie de vértigo de falsificación.

Palabras clave: intertexto, ficción, mentira, traición, traducción

\begin{abstract}
This essay will examine the intertextual relationship between Jorge Luis Borges amd Tahar Ben Jelloun. The Argentinian writer appears as a character in the novel by Ben Jelloun, L'enfant de sable. The inclusion of Borges in the novel contributes to the instability of the narrative world. In Ben Jelloun's novel it is impossible to grasp the veracity of the history that is narrated. The presence of Borges in the story points to an inversion of the values of fiction and reality. The identity of the characters is at stake and the continued narration of stories is their only means to salvation in what appears to be a vertigo of falsification.
\end{abstract}

Key words: intertext, fiction, falsehood, betrayal, translation 
$\mathbf{E}$ n la lectura equivocada que, de acuerdo con Ricardo Piglia, Borges hace del mundo, encontramos una traición recurrente y sistemática en la que el escritor fundamenta su creación. Hacedor de fábulas, su poética de la ficción desestabiliza el mundo al transgredir los límites entre la realidad y la ficción. En ese mundo de representaciones falsificadas, entre uno de los tantos viajes que Borges hace de un cuento a otro, disfrazado de presencia intertextual, fantasma de la fábula a través de siglos inexistentes, llega a habitar el tejido narrativo de Tahar Ben Jelloun como un trovador ciego ávido de historias y, también, de redención. A través de esta red intratextual, L'enfant de sable, el texto de Ben Jelloun, se transforma en espejo que refleja la imagen de un Borges consagrado a una lectura de lo invisible. En el mismo texto, por medio de una alquimia textual, Borges se transforma en transcripción y en objeto; su obra, narrada por un halaquí que inventa una fábula que ya ha sido escrita en los senderos de la biografía, hace circular el zahir y, con él, el fundamento de una serie infinita de mundos posibles, susceptibles de traición.

Identidad trastocada, la mentira instala el juego entre el yo y el otro; entre el texto y su falsificación se abre un espacio en el que tanto las palabras como el falsificador sufren una transformación esencial. El hacedor se ha apropiado de las palabras de otro y, más allá de una transcripción de las mismas, ha modificado su identidad y su memoria. Así, entre la extrañeza y la apropiación, se revela la idea de traducción como una instancia que intenta llenar el espacio vacio que ha dejado la pérdida de la similitud entre la palabra y la cosa. Es el intento por recuperar la unidad perdida, el tiempo anterior a la fragmentación provocada por la torre de Babel; el intento por recobrar la memoria divina, un paraíso que se sabe irrecuperable.

Es necesario traducir porque hay un sentido extraviado, como también es necesario traducir porque hay una identidad y una memoria, configuradas por una tradición a la que es necesario responder; de esta forma, la traducción es un acto de apropiación y diferencia. Para Michel Foucault, la lectura de los signos reconstruye el orden del mundo; en ese mundo, las palabras nos escriben desde el primer día de la creación. Una construcción verbal dibuja nuestro destino; no obstante, se trata de una escritura que debe ser reconstruida y traicionada para tratar de dar forma a una identidad a la que no se ha acabado de dar forma. Tan- 
to Borges como Ben Jelloun hacen referencia a un secreto sin revelar; la única clave para desentrañarlo consiste en la fabulación, en recurrir a la palabra, a la lectura de textos inscritos en los siglos, ya sea la expresión sagrada del Corán, la voz extraviada de los narradores infinitos de las Mil y una noches, o los textos árabes descubiertos por el perseguidor de ficciones en las inscripciones de la mezquita de Córdoba. Como en la tradición cabalística, el secreto debe ser transcrito e interpretado; es necesario reinventar la fabulación que da forma al mundo, apropiarse de ella y, a la vez, traicionarla.

\section{Dioses de ficción}

En el punto en que Borges se inserta en la narración de Tahar Ben Jelloun, queda establecida la ficción como una poética encargada de tejer la biografía del error y la mentira. En los relatos de Borges, un hacedor falsifica la trama de la realidad porque él mismo es un dios erróneo en una sucesión de mundos duplicados, de espacios y de tiempos que se entrecruzan para narrar una historia en la que el final pueda ser repetido hasta el infinito, hasta el momento en que la revelación de una imagen especular advierta que entre la realidad y la fábula puede existir un destino semejante. De acuerdo con Ricardo Piglia: "el arte de narrar es el arte de la percepción errada y de la distorsión. El relato avanza siguiendo un plan férreo e incomprensible y recién el final surge en el horizonte una realidad desconocida: el final hace ver un sentido secreto que estaba cifrado y como ausente en la sucesión clara de hechos" (Piglia, 2000: 124).

Los habitantes de la fábula reconocen su existencia ficcional después de recorrer su propio laberinto; en ese instante, el reconocimiento de su monstruosidad les revela el vértigo de la falsificación. Según Piglia (2000: 124): "los cuentos de Borges tienen la estructura del oráculo: hay alguien que está ahí para recibir un relato, pero hasta el final no comprende que esa historia es la suya y que define su destino". Es la revelación final de Asterión, cuando la espada de bronce de Teseo refleja el sol del amanecer y el Minotauro, atrapado en el laberinto, deja de narrar; confrontado a su destino, el monstruo es destruido por el héroe y, por otro lado, consciente de su presencia mítica, fantasmal, se disuelve ante la revelación de su ficcionalidad: 
Le récit parvient donc à nous faire partager ce point de vue étrange et monstrueux, jusqu'à ce qu'on découvre à la fin le piège narratif. Mais l'intérêt de ce récit n’est pas tant que le narrateur soit un monstre, une infamie matérialisée et cachée, mais qu'il soit un monstre dont le lecteur n'ignore pas qu'il est désormais une pure fiction littéraire. Car le Minotaure est un monstre littéraire de convention, un monstre "de papier" (Lellouche, 1989: $345)$.

En Dreamtigers, el soñador crea un tigre imperfecto que no alcanza una forma acabada: "Nunca mis sueños saben engendrar la apetecida fiera. Aparece el tigre, eso sí, pero disecado o endeble, o de un tamaño inadmisible, o harto fugaz, o tirando a perro o a pájaro" (Borges, 1996d: 161). En la poética del sueño, la traición a la realidad revela el poder ilimitado de la ficción; en la poética del juego, se manifiesta la posibilidad de mentir con la verdad: "una potencia del engaño ocurre en el truco: ese jugador rezongón que le ha tirado sus cartas sobre la mesa, puede ser ocultador de un buen juego (astucia elemental) o tal vez nos está mintiendo con la verdad para que descreamos de ella (astucia al cuadrado)" (Borges, 1966: 146). El engaño, al final, logra desarticular las categorías de verdad y de realidad, provocando un desequilibrio en el que la voluntad de alterar el mundo surge como la copia malvada de un primer demiurgo perdido en el juego de falsificaciones infinitas.

Cuando Borges, traductor de sus ficciones y falsificador de su propia biografía, llega a instalarse como halaquí en la plaza Xmâa el Fna, en la novela de Tahar Ben Jelloun L'enfant de sable, refiere que su presencia en esa plaza se debe al contrabando de palabras hecho por Si Abdel Malek, el narrador principal de la historia de Ahmed: “je ne sais pas quelles mains mont poussé jusqu’à vous. Je crois que ce sont celles de votre conteur qui doit être un contrebandier, un trafiquant de mots" (Ben Jelloun, 1985: 173). Al explicar su procedencia, asegura haber vivido el incendio de un templo circular coronado por un tigre o un caballo de piedra; asegura también que, al descubrir que el fuego no devoraba su carne, el terror y la humillación se apoderaron de él al revelarle que no era sino el sueño de otra apariencia. 
Borges, posible construcción de Si Abdel Malek, transfigurado a través de los relatos y los siglos, testimonia una poética de la ficción en la que los personajes de la fábula amenazan a su creador con la locura y el vacío:

Ce fut durant une de ces nuits que la mort mapparut sous les traits d'un personnage, la huitième naissance, Ahmed ou Zahra, et qui m’a menacé de toutes les foudres du ciel. Il me reprochait d'avoir trahi le secret, d'avoir souillé par ma présence l'Empire du Secret, là où le Secret est profond et caché (Ben Jelloun, 1985: 203).

El halaquí está condenado a cargar con el peso fatídico de una historia inconclusa; lo caminos de la invención han sido cerrados por haber instalado inventado un destino para sus personajes. Rebasado por su creación, debe asumir la maldición que lo persigue en el exilio y lo acerca a las sombras de la muerte; así, Fatima, la que fuera esposa de Ahmed en un doble juego de falsificación, el de la ficción y el del cambio de identidad, dice: "je reviens sur les pas de ton histoire. Je compte les morts et jattends les survivants. Tu ne peux rien contre moi. J'appartiens à cette éternité dont tu parles sans la connaître" (Ben Jelloun, 1985: 205).

Desde ese espacio de eternidad, Ahmed, transfigurado en Zahra, visita al trovador ciego en su guarida de la biblioteca de Buenos Aires:

je me dis, à force d'inventer des histoires avec des vivants qui ne sont que de morts et de les jeter dans des sentiers qui bifurquent ou dans des demeures sans meubles, remplies de sable, à force de jouer au savant naïf, voilà que je suis enfermé dans cette pièce avec un personnage ou plutôt un énigme, deux visages d'un même être complètement embourbé dans une histoire inachevée, une histoire sur l'ambiguité et la fuite! (Ben Jelloun, 1985: 179).

Como Asterión, el trovador está atrapado en un laberinto; como en el relato de Borges, el trovador reconoce que hay otro, también criatura de la falsificación, capaz de destruirlo. Entre Zahra y él se interpone el Corán, el libro sagrado que puede evitar la mentira; sin embargo, las manos de ambos tocan simultánea- 
mente otro libro esencial: el Quijote; entonces, la mujer misteriosa dice al trovador que no busca la verdad sino el olvido, la posibilidad de borrar su pasado y de escapar de la simulación:

ce que je cherche, c'est n'est pas la vérité. Je suis incapable de la reconnaitre. Ce n'est pas la justice non plus. Elle est impossible. Il y a dans ce Livre des versets qui ont la fonction de loi; ils ne donnent pas raison à la femme. Ce que je cherche, ce n'est pas le pardon, car ceux qui auraient pu me le donner ne sont pas là. Et pourtant j’ai besoin de justice, de vérité et de pardon. (Ben Jelloun, 1985: 181).

Ahmed-Zahra desaparece llevándose la novela de Cervantes. El trovador ciego ya no podrá escapar del laberinto propuesto por el retablo de las maravillas. Comprometido para siempre con la ficción, sabe que no puede escapar de ella porque su propia existencia está en juego. De Zahra y el trovador ciego, de Borges y de Si Abdel Malek, puede decirse lo mismo que afirma la Parábola de Cervantes y de Quijote: "no sospecharon que los años acabarían por limar la discordia, no sospecharon que la Mancha y Montiel y la magra figura del caballero serían, para el porvenir, no menos poéticas que las etapas de Simbad o que las vastas geografías de Ariosto" (Borges, 1996d: 177). En el interior del libro, o del secreto, tiene lugar la falsificación. Borges y Si Abdel Malek, hacedores de trucos, como los jugadores de cartas de Evaristo Carriego, al instalar la falsedad construyen la hipótesis de un mundo. De acuerdo con Lellouche (1989: 351):

nous sommes au cœur de la question de lêtre feint, de lêtre-de-fiction, nos pas seulement comme un être fictif, mais comme être supposé. Non pas seulement comme semblant prodigué, mais comme suppôt. Le magicien des Ruines circulaires veut « feindre » un homme, et se reconnait feint luimême, «supposé » par un Autre, nous-même ou Borges, ou Dieu. 


\section{La traición}

Según Foucault, los signos presentes en la naturaleza han sido dispuestos por Dios para ser descifrados: "le langage n'est pas un système arbitraire; il est déposé dans le monde et il en fait partie à la fois parce que les choses elles-mêmes cachent et manifestent leur énigme comme un langage, et parce que les mots se proposent aux hommes comme des choses à déchiffrer (Foucault, 1966: 50). En el comienzo del mundo, Dios establece una equivalencia perfecta entre las palabras y las cosas y, así como las características de los elementos de la naturaleza están inscritas en sus nombres, la influencia de los astros está marcada en el cuerpo de los hombres. Pero después de la turbulencia de Babel, se produce una desgarradura entre el hombre y lo divino, entre la palabra y el objeto. Por su parte, Steiner (2006: 41) afirma : "la Chute de l'homme (de la vie humaine) est celle de la présence réelle du signifiant vers la re-présentation [...] dans le signe”.

La lengua se convierte en presencia exiliada; el exilio, producto de la fragmetación de la unidad divina, forma parte de la condición humana y, con ello, la necesidad de traducir:

même quand elle est la plus attentive aux phénomènes, la plus tendue vers la vérité, la langue orale ou écrite des hommes et des femmes après l'Eden est conjecturale et spéculative. Cette abrogation de l'immédiateté est propre a chaque langue. Ce qui nécessite à son tour toute larmature de la traduction et de la mé-traduction au sein d'une matrice monolingue (Steiner, 2006: 41).

La desgarradura inherente a la lengua transforma en secreto la transparencia con que reflejaba el mundo. Una vez que los signos han velado su discurso, es necesario interpretarlos:

le langage est à mi-chemin entre les figures visibles de la nature et les convenances secrètes des signes ésotériques. C’est une nature morcelée, divisée contre elle-même et altérée qui a perdu sa transparence première ; c'est un secret qui porte en lui, mais, à la surface, les marques déchiffrables de ce qu'il veut dire (Foucault, 1966: 50). 
Al revelar su capacidad para representar, el lenguaje, después de Babel, comienza a mostrarse como espacio lúdico en el que la transformación de los objetos a través de la interpretación de los signos se vuelve posible. Pero, por otra parte, Babel también es el comienzo de la traición, el momento en el que, al apropiarse del secreto para trasladar su sentido, se transforma y falsifica; en este sentido, Steiner escribe: "toute communication intralinguistique est, au fond, traduction. Le signe doit toujours être négocié, avec ce que cela suppose de résidu ou de redondance, de malentendu ou de paraphrase" (Steiner, 2006: 42).

La ficción revela la conciencia de la mentira al constituirse como espacio lúdico, juego de reconstrucción y de engaño en el que la identidad de los participantes está siempre en riesgo. En el juego de la creación de mundos posibles, las palabras representan, interrogan y falsifican, como escribe Borges en "El truco", uno de los episodios de Evaristo Carriego:

la trucada se arma; los jugadores, acriollados de golpe, se aligeran del yo habitual. Un yo distinto, un yo casi antepasado y vernáculo, enreda los proyectos del juego. El idioma es otro de golpe. Prohibiciones tiránicas, posibilidades e imposibilidades astutas, gravitan sobre todo decir. Mencionar flor sin tener tres cartas de un palo, es hecho delictuoso y punible, pero si uno ya dijo envido, no importa. Mencionar uno de los lances del truco es empeñarse en él: obligación que sigue desdoblando en eufemismos a cada término. Quiebro vale por quiero, envite por envido, una olorosa o una jardinera por flor (Borges, 1996a: 145).

En el escenario de la ficción, la representación de un mundo nos lleva a las trampas de la representación; el hacedor, como Si Abdel Malek y el trovador ciego, falsifica historias para crear universos más diabólicos que la realidad, como dice el narrador de Evaristo Carriego; sin embargo, al igual que el jugador, "no hace ya más que reincidir en bazas remotas. Su juego es una repetición de juegos pasados, vale decir, de ratos de vivires pasados" (Borges, 1996a: 146).

La traducción, avatar del palimpsesto, regresa a una memoria fijada por la voz de otro. En un primer momento, el trovador ciego afirma que la historia de Ahmed le interesa por no ser una traducción de la realidad; el trovador reconoce así su filiación falsificadora: "je la prends telle qu’elle est, artificielle et 
douloureuse" (Ben Jelloun, 1985: 172). Pero Si Abdel Malek anuncia que esta historia ha sido escrita por el propio Ahmed y, sólo al final, sabemos que hay un relato original, procedente de Alejandría, que la tía de Bey Ahmed entregó al halaquí. Éste, el mejor narrador de la plaza, acepta el reto y decide insertar la historia en el mundo marroquí; sin embargo, traiciona el destino del personaje modificando los detalles de su vida.

Si Abdel Malek falsifica y traiciona; a partir del personaje real, construye una entidad ficcional que, posteriormente, se escapa de su dominio. El narrador traduce una serie de formas culturales, elabora la lectura de una tradición y decide reconstruirla, aunque, como fabulador, ha tenido que falsificar el relato original. En este sentido, podemos reconocer el planteamiento de Steiner, quien reconoce la cultura como una traducción de formas y de significados heredados.

En el proceso que relaciona la traducción con la memoria, en la historia de Ahmed se presenta una interesante relación entre traducción, tradición e identidad. La traducción, la lectura y la escritura serían las manifestaciones de una memoria que, "a modo de palimpsesto, se inscriba en cada cosa como una herencia continua que habrá de desmembrarse y que se revela en la palabra como acontecimiento" (Garza, 2006: 46).

En relación con la identidad falsificada de Ahmed, quizás el primer ejemplo de traducción como traición está presente en que el padre de éste anuncia su nacimiento en el periódico nacional; el texto significa el comienzo de la simulación al señalar la identidad masculina de Ahmed a través del nombre: "un garçon -que Dieu le protège et lui donne longue vie -est né jeudi à $10 \mathrm{~h}$. Nous l'avons nommé Mohamed Ahmed" (Ben Jelloun, 1985: 30). El cambio de la identidad del personaje representa una traducción violenta de su identidad, a la que va a oponerse hasta el final.

En su supuesto diario, Ahmed relata cuando iba a la mezquita con su padre y, mientras escuchaba los cantos coránicos, veía las inscripciones árabes grabadas en el techo y, en algunos casos, indica la traducción de dichas inscripciones; en otro momento, describe cómo se deja llevar por la musicalidad del alfabeto: “je m’accrochais au Alif et me laissais tirer par le Noun qui me déposait dans les bras du Ba. J'étais ainsi pris par toutes les lettres qui me faisaient faire le 
tour du plafond [...]"(Ben Jelloun, 1985: 38). Al igual que Ahmed, el trovador ciego escucha la recitación del alfabeto árabe en los jardines de la Alhambra.

Ahmed, consciente de la esencia poética de su lengua, se apodera de ella para tratar de recobrar su identidad; por esta razón, cuando su madre lo lleva al hamam, escucha las palabras que dicen las mujeres, que quedan adheridas en el techo, como si éste fuera un tablero de escritura; después, las palabras descienden convertidas en gotas de agua. Pero las palabras prohibidas para las mujeres, aquellas que se refieren al sexo, permanecen en el techo, y sólo una vez, cuando Ahmed escucha los insultos de dos mujeres, se decide a guardarlas en su cuerpo: "lorsque ma mère me savonnait, elle était étonnée de constater combien jétais sale, et moi je ne pouvais pas lui expliquer que le savon qui coulait emportait toutes les paroles entendues et accumules le long de cet après-midi” (Ben Jelloun, 1985: 35, 36). Las palabras le permiten tomar conciencia de su cuerpo, más allá de la voluntad de falsificación impuesta por la familia; al sobrescribir las palabras en su piel, está haciendo una lectura y una interpretación de su cuerpo y de su identidad, en un afán por encontrar la verdad.

Ahmed va en contra de la falsificación que le han impuesto; su recurso más importante se encuentra en las palabras, ya sea a través de la lectura, o de la escritura de su diario; en cualquier caso, las palabras son la entidad de la que se vale para llevar a cabo la interpretación de su identidad. La traducción es metáfora de la búsqueda; en Córdoba, Zahra encuentra al trovador ciego con una traducción del Corán; en Buenos Aires, la misma mujer lee todas las traducciones de Las mil y una noches, de donde parece haber surgido, con la voz de Tawaddud. Por su parte, Borges, en "Los traductores de las 1001 noches", se refiere al momento en que los copistas deciden agregar hechos históricos, una vez que la magia ha llegado a su fin; entonces, todo puede estar integrado en un universo de asombros: "las antesalas se confunden con los espejos, la máscara está debajo del rostro, ya nadie sabe cuál es el hombre verdadero y cuáles sus ídolos. Y nada de eso importa; ese desorden es trivial y aceptable como las invenciones del entresueño" (Borges, 2006c: 412).

Zahra entrega al trovador el relato de un sueño; el relato comienza con la transcripción de un poema que atribuye a Firdoussi, un poeta del siglo X. Zahra se identifica con el poema y, en el proceso de transcripción, lo modifica para ex- 
presar su tristeza: "dans ce corps clos, il est un visage éteint, / une blessure, une ombre, et un tumulte, / un corps dissimulé dans un autre corps..." (Ben Jelloun, 1985: 190). En un acto de reconocimiento, Ahmed-Zahra falsifica la escritura de otro, altera la palabra para apropiarse de ella desde su cuerpo y su vivencia.

Por otra parte, el trovador ciego finaliza su intervención con la traducción de un poema de Almoqtâdir El Maghrebi, que vivió en el siglo XII; después de recitar el poema en español, dice la traducción en francés; a diferencia de Ahmed-Zahra, el trovador no altera las palabras del poema, que al final dice: "¿es posible que yo, súbdito de Yaqub Almansour, / Muera como tuvieron que morir las rosas y Aristóteles?" (Ben Jelloun, 1985: 198). Sin embargo, aunque un momento antes el trovador habla de la cercanía de la muerte, niega identificarse con el recitante, como si quisiera separarse del universo que acaba de enunciar. En el primer caso, se pone de manifiesto la identificación con un texto traicionado, mientras que, en el segundo, hay una distancia que va del recitante al poema, traducción literal de su original. Es claro que se trata de dos maneras distintas de asumir la traducción, ya sea como apropiación de la traición, o como distanciamiento frente a la fidelidad; en ambos casos, se produce un juego entre el yo y el otro, entre la copia y la transformación de la que habla Borges en Pierre Menard:

no quería componer otro Quijote - lo cual es fácil— sino el Quijote. Inútil agregar que no encaró nunca una transcripción mecánica del original; no se proponía copiarlo. Su admirable ambición era producir unas páginas que coincidieran - palabra por palabra y línea por línea- con las de Miguel de Cevantes (Borges, 1996b: 444).

La traducción como traición deja entrever un mundo supuesto; la hipótesis de la creación en la que el hacedor puede ser una ficción. En el caso de Borges y el de Ben Jelloun, la obra ficticia forma parte de un juego de espejos que da cuenta de la infinitud de la invención. El mundo no se cierra porque las posibilidades de interpretación, de reescritura y de falsificación siguen realizándose a través de un número ilimitado de demiurgos. Como la biblioteca de Babel, que contiene todas las posibles combinaciones de signos, abriéndose al vértigo del vacío, el diario de Ahmed es una sucesión de páginas en blanco dispuestas a ser 
grabadas por múltiples escrituras. Borges se refugia en el abismo de la ficción, mientas Si Abdel Malek lee el Corán entre las tumbas. Tanto la palabra ficticia como la palabra sagrada se inscriben en el universo para ser descifradas, aunque se muestren siempre inalcanzables.

La referencia a un mundo traicionado por los mecanismos de la ficción manifiesta que la reconstrucción de la realidad implica una transformación de la identidad. La idea de traducción como proceso de transformación nos conduce a la noción de traición en la medida en que el desciframiento de un universo implica una transfiguración y, en muchos casos, una falsificación.

La presencia de Jorge Luis Borges en la obra de Ben Jelloun establece una poética de la ficción en la que la falsificación representa el papel fundamental. Si bien es cierto que hemos reconocido las formas en que dicha presencia se manifiesta, ha sido necesario desentrañar el efecto que esa inserción produce en el texto de Ben Jelloun. Al reconocer la falsedad como el eje en que se centra la narración de L'Enfant de sable, encontramos que el punto de encuentro entre la ficción de Borges y la de Tahar Ben Jelloun radica en la concepción de la ficcionalidad como un universo de interpretaciones infinitas que ponen en riesgo la identidad de los habitantes de dicho universo, que, a su vez, se revela como estructura portadora de un secreto que debe ser descifrado a través de múltiples lecturas, de traducciones y reconstrucciones que, no obstante, no otorgan un sentido completo de la creación. La traición de la realidad propone la hipótesis de un mundo cuyos habitantes se han convertido en espejismo, que no puede ser verdadero si es ajeno. En este caso, la traducción permite una apropiación del mundo, así como la posibilidad de la búsqueda de claves de su desciframiento; como afirma Piglia:

el narrador se refugia nuevamente en la lectura; en otro tipo de lectura esta vez, una lectura controlada, minuciosa, la lectura como traducción. El traductor es aquí el lector perfecto, un copista que escribe lo que lee en otra lengua, que copia, fiel, un texto, y en la minuciosidad de esa lectura olvida lo real (2005: 29). 


\section{Referencias bibliográficas}

BEN JELLOUN, Tahar (1985): L'enfant de sable. París: Seuil.

BENJAMIN, Walter (2000): "La tâche du traducteur". En Euvres I (trad. de Maurice de Gandillac, Rainer Rochlitz y Pierre Rusch). París: Gallimard.

BORGES, Jorge Luis (1996a): "Evaristo Carriego". En Evaristo Carriego. Obras completas. Vol. I, Buenos Aires: Emecé.

BORGES, Jorge Luis (1996b): Pierre Menard, autor del Quijote", "Las ruinas circulares". En Ficciones. Obras completas. Vol. I, Buenos Aires: Emecé.

BORGES, Jorge Luis (1996c): “Los traductores de las 1001 noches”. En Historia de la eternidad. Obras completas. Vol. I, Buenos Aires: Emecé.

BORGES, Jorge Luis (1996d): “Dreamtigers”, "Parábola de Cervantes y de Quijote”. En El hacedor. Obras completas. Vol. II, Buenos Aires: Emecé.

FOUCAULT, Michel (1996): Les mots et les choses: une archéologie des sciences humaines. París: Gallimard.

GARZA SALDÍVAR, Norma (2006): "La memoria del palimpsesto". En Metapolítica. Vol. 10, núm 47, pp. 46-50.

LELLOUCHE, Raphaël (1989): Borges ou l'hypothèse de l'auteur. París: Balland.

PIGLIA, Ricardo (2005). El último lector. Barcelona: Anagrama.

PIGLIA, Ricardo (2000): Formas breves. Barcelona: Anagrama.

STEINER, George (1995): Después de Babel: aspectos del lenguaje y la traducción. México: FCE.

STEINER, George (2006): "De la traduction comme 'condition humaine'”. En Magazine Littéraire. Núm. 454, pp. 41-43. 